\title{
Abnormal gait detection by means of LSTM
}

\author{
César G. Pachón-Suescún, Javier O. Pinzón-Arenas, Robinson Jiménez-Moreno \\ Faculty of Engineering, Nueva Granada Military University, Bogotá D.C., Colombia
}

\section{Article Info}

Article history:

Received Sep 18, 2019

Revised Oct 11, 2019

Accepted OCt 20, 2019

Keywords:

Abnormal gait patterns

Kinect sensor

LSTM

\begin{abstract}
This article presents a system focused on the detection of three types of abnormal walk patterns caused by neurological diseases, specifically Parkinsonian gait, Hemiplegic gait, and Spastic Diplegic gait. A Kinect sensor is used to extract the Skeleton from a person during its walk, to then calculate four types of bases that generate different sequences from the 25 points of articulations that the Skeleton gives. For each type of calculated base, a recurrent neural network (RNN) is trained, specifically a Long shortterm memory (LSTM). In addition, there is a graphical user interface that allows the acquisition, training, and testing of trained networks. Of the four trained networks, $98.1 \%$ accuracy is obtained with the database that was calculated with the distance of each point provided by the Skeleton to the Hip-Center point.
\end{abstract}

Copyright (C) 2020 Institute of Advanced Engineering and Science. All rights reserved.

Corresponding Author:

Robinson Jiménez Moreno,

Mechatronics Engineering Program, Faculty of Engineering,

Nueva Granada Military University,

Carrera 11 \#101-80, Bogotá D.C., Colombia.

Email: robinson.jimenez@ unimilitar.edu.co

\section{INTRODUCTION}

The design of systems based on artificial intelligence techniques with a focus on medicine has gained importance in various research. In [1], it was sought to automate the detection of multiple sclerosis, implementing deep learning techniques, specifically the convolutional neuronal networks (CNN), where a correlation of 0.97 is obtained with respect to the manual annotations of the white matter, concluding that the use of $\mathrm{CNN}$ to automate processes of detection of white matter is close to that of experts, being a clear starting point for the implementation of this type of systems.

$\mathrm{CNN}$ is a Deep learning technique [2], that has been demonstrated its high performance in pattern recognition [3], with an emphasis on image classification [4]. This technique has been to have some evolutions in complementaries architectures like the DAG-CNN [5] and the R-CNN [6]. In [7], CNNs are used for segmentation of magnetic resonance images, in order to classify images of brains according to the age of the person, where $82 \%$ to $91 \%$ of the precision of the classifications is obtained.

In [8], it is emphasized that with current methods, the diagnosis of Parkinson's disease tends to be subjective, for this reason a Kinect sensor [9] is used as a support for the diagnosis of Parkinson's, seeking to capture by means of this, the movement of patients, the movement of patients, extracting characteristics such as amplitude and frequency of movement, which are important for physicians to make a correct diagnosis of this disease. In [10], it is mentioned that currently for the detection and identification of neurodegenerative diseases, physicians tend to analyze the progress of patients, in this case, they focus on the detection of Parkinson's disease, Huntington and amyotrophic lateral sclerosis, implementing a type of RNN called LSTM, achieving a $95.67 \%$ accuracy in the identification of the gaits.

Several studies have already focused on the analysis of alterations in the gait, behavior or postures of people. In [11], a type of shoes were designed that are made up of various sensors such as load cells, accelerometers, and gyroscopes that help to identify the gait of the person, vectors of machine supports 
(SVM) are implemented for the classification between types of normal gait, foot inwards, foot outwards, among others, obtaining between $89.6 \%$ and $93.38 \%$ accuracy in the classification of gait abnormalities, presenting as a disadvantage that the methods used tend to be intrusive for the user. In [12], different types of RNN are evaluated, such as the LSTM, the vanilla RNN, and the Gated Recurrent Unit GRU, to detect abnormal behaviors in elderly people with dementia, it is concluded that the LSTM has a better behavior achieving up to $96.7 \%$ accuracy in the identification of abnormalities. In [13], using CNN and the movement of the Skeleton of a person turn it into an image to detect the actions that are being carried out, such as movements in the body and actions in sports, the proposed method is tested with different databases, obtaining percentages of accuracy between $86.97 \%$ and $95.45 \%$.

For the recognition of human actions, it becomes recurrent in current works, the implementation of deep learning algorithms whether through CNN or LSTM, demonstrating how robust and precise these techniques can be. As an example of the above in [14], genetic algorithms (GA) and CNN are used to recognize among 50 human actions of the UCF50 database [15], the GAs are used to initialize the CNN weights, achieving a 99.98\% accuracy in the classification. In [16] and [17], the LSTM is used for the recognition of actions in people, where the first one focuses on identifying the action that a person is performing (walking, sitting, standing, jogging, among others), achieving $92.1 \%$ accuracy in the identification of actions. While in the second one, the use of LSTM is highlighted for the analysis of actions in a spatio-temporal way, the method is tested in different databases, obtaining a $95 \%$ accuracy in the classification of the UT-Kinect database [18].

The World Health Organization (WHO), conducted a study of neurological disorders and the challenges they presented to public health, where a forecast was made which mentioned that by 2030, $12.22 \%$ of deaths would be attributed to neurological disorders [19]. These studies demonstrate the need to focus research on the identification and detection of neurological diseases. For this reason, this article exposes an investigation oriented in the detection and identification of three types of abnormal patterns in gait that are caused by some neurological disease: Parkinsonian gait, Hemiplegic gait, and Spastic Diplegic gait.

The aim is to detect gait abnormalities by implementing LSTM. In [20], besides explaining the general operation of the LSTM, it is mentioned that they are a special type of RNN, that do not present the problem of long-term dependence that the classic RNNs do, which are explained in depth in [21], thus avoiding the problems that arise in the gradients of the deeper gradient of the network. This type of network was presented in [22], where it is emphasized that the implementation of LSTM leads to more precise results and faster training, compared to recurrent learning and retro-propagation over time.

The paper is divided into 7 parts. In the first part, the general outline of the process is presented. The second focuses on the acquisition of the database and the calculation of input characteristics for section three, which focuses on the architecture of the LSTM and its training. In the fourth part, some of the cases of correct and incorrect classification of the network are shown. The fifth part presents a graphic user interface designed to facilitate and automate the process of identifying the type of gear. Then the classification times of one of the trained networks are presented. Finally, conclusions are given on the results obtained in each of the stages.

\section{RESEARCH METHOD}

\subsection{Process scheme}

Figure 1 shows the general process of the proposed method to detect the type of gait abnormality that a person may have. The initial step is to perform the acquisition of the person's gait, for this it is decided to use a Kinect V2, taking into account that it is a non-intrusive system and therefore it will not affect or cause variations in the patients' walk during the tests. In addition, it has already been implemented in similar research as evidenced in the state of the art. The user must walk in front of the sensor, taking into account that his whole body must be seen so that the Skeleton can be extracted correctly. Once it is detected that there is no one in the field of vision of the sensor, a simulation of the person's walk is generated, using the extracted Skeleton, it should be noted that each frame of the video has a resolution of 512x424 pixels. From the coordinates of each point of the Skeleton in each of the tables of the video, the sequences that will be entered into the LSTM are calculated. Finally, the network classifies the gait entered into 4 categories (normal gait, Spastic Diplegic gait, Hemiplegic gait, and Parkinsonian gait). 


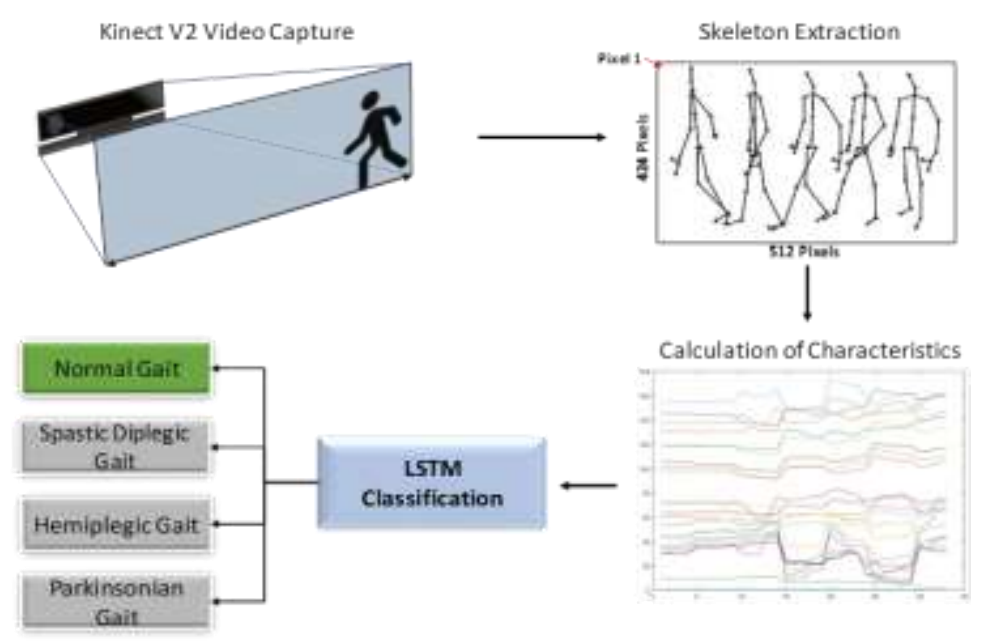

Figure 1. Process scheme

\subsection{Database}

The stage of the database begins with the analysis of the data that the Kinect sensor provides from the person. Then it is necessary to understand the main characteristics of each of the gaits to be classified. Subsequently, four different types of databases are generated, with each of these a network will be trained. Finally, the number of training sequences and tests for the network is established.

\subsubsection{Kinect data of the Skeleton}

In Figure 2, each of the joints that can identify a person, the Skeleton function of Kinect, being 25 in total. What the Skeleton provides are the coordinates of each of these points in each captured frame. With these coordinates, each of the joints can be linked to visualize the skeleton of the person in his trajectory. Only 5 frames per second are captured, being enough to obtain different positions of the body during the march.

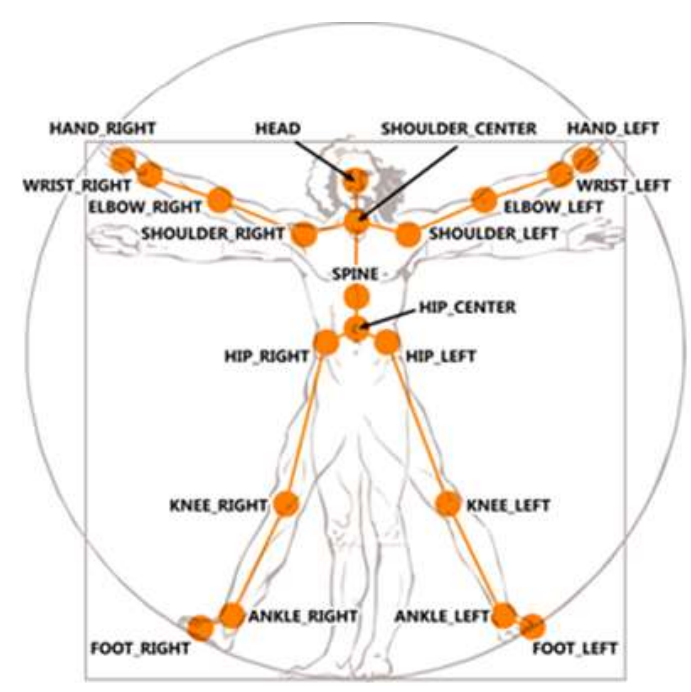

Figure 2. Joints to form the skeleton [4]

\subsubsection{Gait patterns acquired}

This project is the first approach to evaluate the potential of LSTM to detect different abnormalities in gait patterns, for the acquisition of the database were used people who did not suffer any abnormality. Therefore, to generate the database, it is necessary to understand the main features of each one of the gait patterns, in order to imitate them correctly during the acquisition of the data. In [23, 24], each of the gait 
abnormalities caused by some type of neurological problem is explained in detail. In general, the Spastic Diplegic gait, characterized by paralysis of the upper or lower extremities, is usually caused by cerebral palsy, the patient tends to walk with the upper part of the legs narrowly and shuffle when walking. In the Hemiplegic gait, unlike the previous one, the paralysis only occurs in one part of the body, the arm is flexed and rotated internally, the leg of the same affected area is in extension and when walking, semicircles are made with that leg. Finally, the Parkinsonian gait is characterized because the patient is with knees bent, the head bent and advances with small steps.

In total, 220 videos are acquired for the database that meet the characteristics of each gait pattern to be evaluated, 55 videos per each type of gait, each representing one-quarter of the total database. Figure 3 shows an example of each of the four marches to be classified in the network, it should be noted that for reasons of correct visualization, only the most representative Skeleton frames are shown during the trajectory.
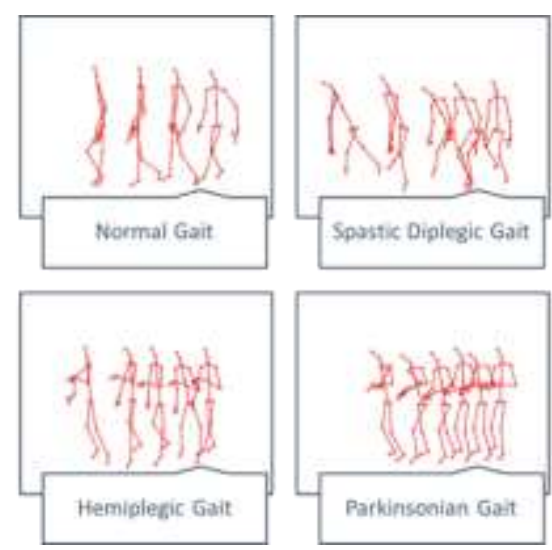

Figure 3. Gait patterns

\subsubsection{Calculation of databases}

The base information used corresponds to the pixel coordinates of the $\mathrm{X}$ and $\mathrm{Y}$ axes of each of the 25 points in each frame captured by video. What is sought is to generate databases where their values are representative in the walk of a person, for this reason, 4 different bases are proposed. With each of these bases the network will be trained, and different networks will be compared to verify which method provides greater precision in the classification of walking patterns.

In Figure 4, the graph of the first database is presented, this corresponds to the $\mathrm{X}$ coordinate in pixels of each of the points in each frame of the image where the skeleton was detected. The person starts walking from right to left, this can be evidenced by observing the decrease in the value of the X coordinate as each picture acquired advances. The second base corresponds to the $\mathrm{Y}$ coordinate in pixels in each frame captured where the skeleton was detected as shown in Figure 5. This shows how the Y position of each of the points does not tend to vary significantly in each captured frame.

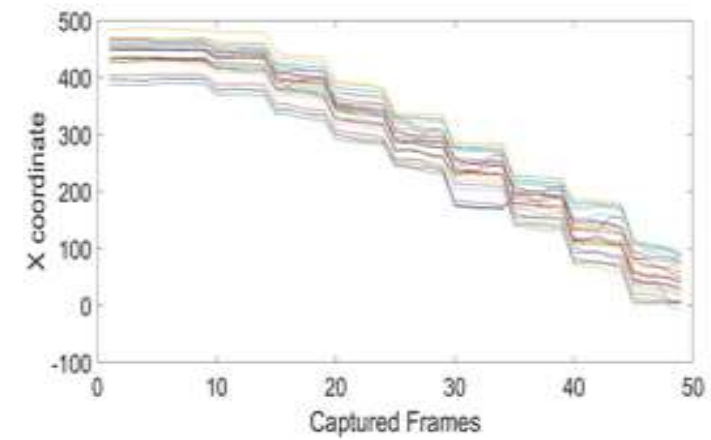

Figure 4. Features of the coordinate $\mathrm{X}$ database

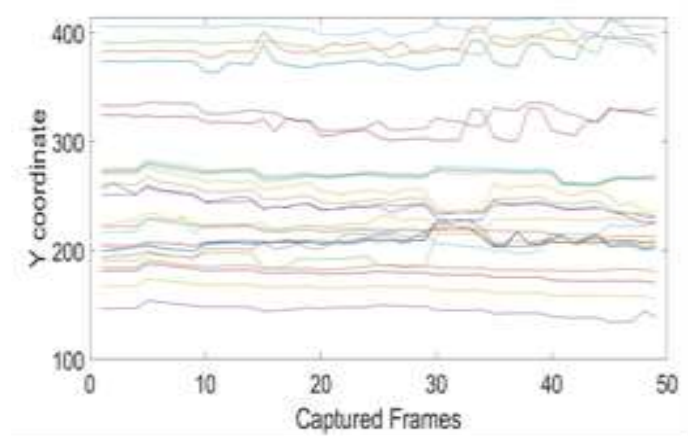

Figure 5. Features of the coordinate Y database 
The third base is calculated with the distance from each of the 25 points of the skeleton to the pixel one of the image, where $X_{a}$ and $Y_{a}$ refer to the current coordinates of the 25 points (1).

$$
D P=\sqrt{\left(X_{a}-1\right)^{2}+\left(Y_{a}-1\right)^{2}}
$$

In Figure 6, the sequence generated by the previous calculation is shown. It can be analyzed from the generated graph, how the distances to pixel 1 of the image with respect to each of the 25 points decreases with each captured frame where the skeleton was detected, this is because the gait is made from right to left.

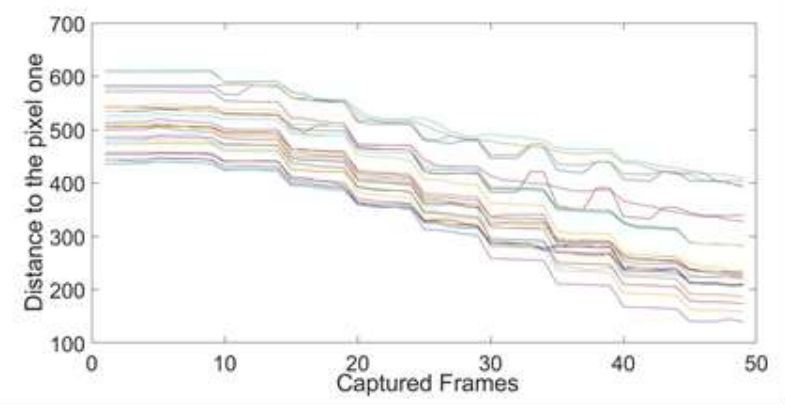

Figure 6. Characteristics of the database from distance to pixel one (DP)

The last database focuses on having a point of reference in the body of the person, specifically the Hip Center presented in Figure 2, and is calculated with the distance that exists from each point of the body to this, where $X_{h c}$ and $Y_{h c}$ refer to the current Hip Center coordinate (2).

$$
D C B=\sqrt{\left(X_{a}-X_{h c}\right)^{2}+\left(Y_{a}-Y_{h c}\right)^{2}}
$$

In Figure 7, the sequence generated by the previous calculation is shown, showing the distance from each point of the body to the Hip Center in each of the captured frames. For this example, a parkinsonian gait is presented.

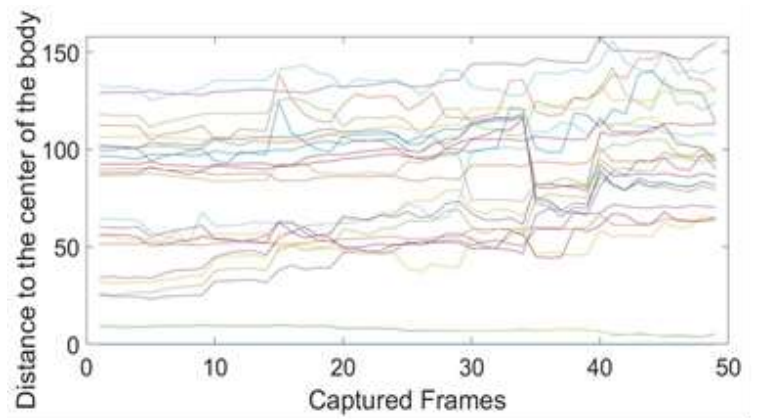

Figure 7. Characteristics database of the distance to the Hip-center (DCB)

\subsubsection{Training and test data}

As input to the network, the sequences of each video are entered according to the database with which it will be trained, highlighting that there will be 25 characteristics in each of the databases. It is established that of the 220 videos captured, 168 are used for network training, corresponding to $76.36 \%$ of the total base and the remaining 52 corresponding to $23.63 \%$ are for test. In Figure 8 , an example of the input sequences for training and testing the network with the DCB database is shown. Where each of the bars represents a sequence of some of the types of gear calculated in the base DCB. In this way, the X, Y, and DP databases have their input sequences to train and test each of the networks. 


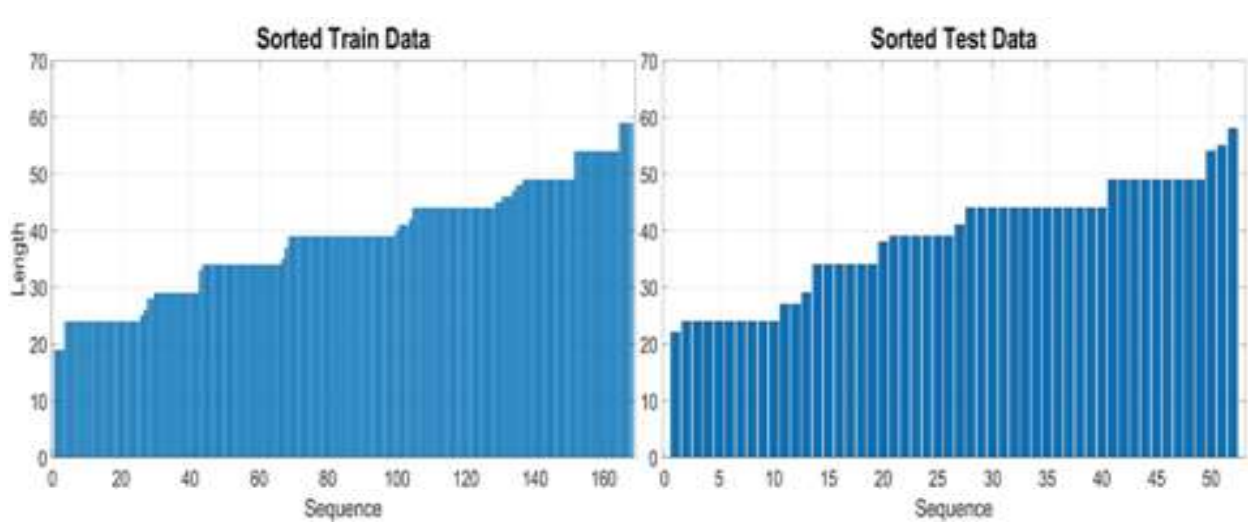

Figure 8. Inputs for training and testing the network

\subsection{Architecture and training}

In Figure 9, the architecture implemented for the network is shown. This architecture is chosen based on an iterative process where parameters such as the number of hidden units, depth of the network and number of Fully Connected layers were varied, looking for the results after training to have a greater percentage of accuracy. Initially, the network receives as input sequences with 25 features, as explained in the stage of creation of the databases, these features will depend on the database with which it is trained or put to the test (base X, Y, DP or DCB). The second layer corresponds to the LSTM, establishing 70 hidden units, finding that with this value a better performance in the training is obtained. Then follows a Fully Connected layer, where the number of filters is 4 corresponding to the number of categories for which the network was trained. Finally, there is the Softmax Function, which is responsible for establishing in each of the output categories the probabilistic value that the input has of belonging to a specific category, where the sum of these values will be 1, in [25] the operation of the softmax is explained in more detail. The category that has the highest value at the exit will be the category in which the network classified the input sequence.

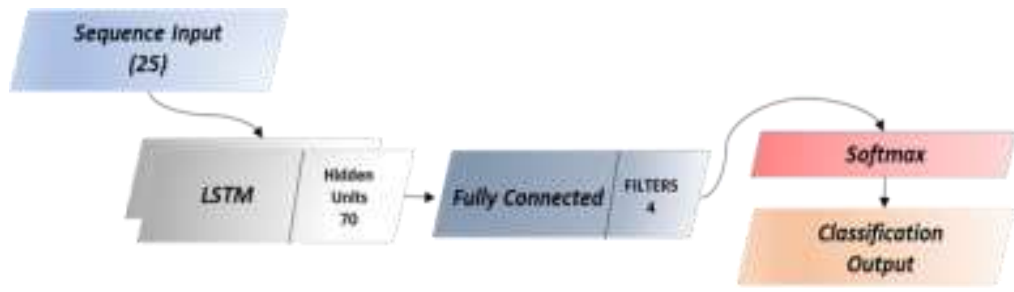

Figure 9. Architecture implemented

For the training of the 4 networks, the same conditions are set. It was observed in the network training graph that with 150 epochs it is enough to obtain adequate percentages of accuracy in the recognition of the type of gait and without overtraining. An initial learning factor (LR) of 0.0001 is set from epoch 1 to 90. In order for the weights to vary less aggressively and to be established in the system minimums, the LR is reduced by a factor of 10 , leaving 0.00001 from 90 to 150 . Finally, the size of the batch is set to 1 , using a small value in this parameter will allow a greater generalization in the training of the network, as indicated in [26].

\section{RESEARCH METHOD}

Table 1 shows a comparison between the 4 networks trained for each of the databases. For each one, the classification accuracy of the sequences in each category is observed, where the Y Database had the lowest accuracy and the DCB database the highest, with a 98.1\% accuracy. The network trained with the DCB database obtained 100\% accuracy in the classification of the Spastic Diplegic, Hemiplegic and Parkinsonian gait sequences. In the normal gait presented a $92.3 \%$ accuracy, incorrectly classifying only one 
sequence, confusing it with a gait type Spastic Diplegic. Figure 10 shows the confusion matrix of the network that obtained the highest precision during training and tests. In the green diagonal are the sequences that were correctly classified, where the last value, corresponding to the lower right box, shows the accuracy obtained by the network, of $98.1 \%$. The rows correspond to the classes that the network predicted and the columns to the real class.

Table 1. Results of the networks trained with each database.

\begin{tabular}{ccccc}
\hline & $\mathrm{X}$ & $\mathrm{Y}$ & $\mathrm{DP}$ & $\mathrm{DCB}$ \\
\hline Normal & $100 \%$ & $100 \%$ & $100 \%$ & $92.3 \%$ \\
Spastic Diplegic & $38.5 \%$ & $0 \%$ & $0 \%$ & $100 \%$ \\
Hemiplegic & $76.9 \%$ & $100 \%$ & $38.5 \%$ & $100 \%$ \\
Parkinsonian & $69.2 \%$ & $0 \%$ & $100 \%$ & $100 \%$ \\
Total & $71.2 \%$ & $50 \%$ & $59.6 \%$ & $98.1 \%$ \\
\hline
\end{tabular}

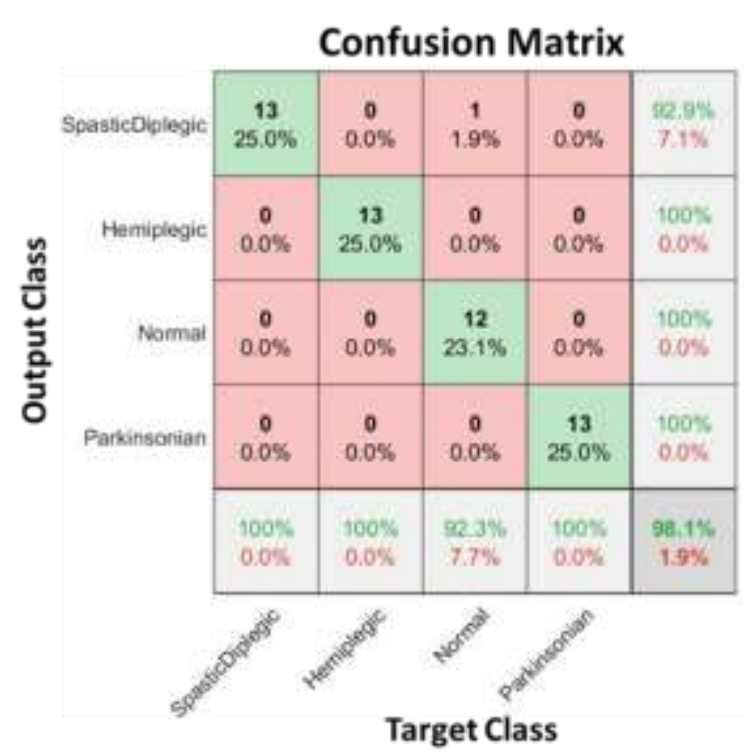

Figure 10. Confusion matrix with DCB database

\subsubsection{Correct and incorrect classifications}

For this stage, only the trained network with the DCB database is analyzed, that is the one that obtained the best performance and therefore it is important to know in more detail what could be the possible causes of the $1.9 \%$ error in this network. Figure 11 shows the sequence of a case of each type of gait that was correctly classified by the network. First, the Hemiplegic Gait is presented, this has as a general feature variation in the distance of the points of the leg that suffers the paralysis with respect to the Hip Center. The normal gait, in general, does not tend to present drastic changes in the distance of each of the points to the center of the body. The Spastic Diplegic Gait is similar to the Hemiplegic gait, with the difference that in this the variations are presented in the features that correspond to both legs. Finally, in the Parkinsonian Gait, as the position of the body that tends to be bent, the features are closer together and given the tremors in the body alterations are shown in some of the characteristics.

In general, the sequences of the gait patterns are clearly differentiated from the DCB base, which allowed the network to learn the patterns of the features. A particular case was classified in the incorrect category, Figure 12 shows a normal walking pattern, which was classified in the spastic diplegic category. If the features of the sequence are analyzed, it can be observed that the beginning of the gait, compared to normal walking, had a behavior that is not characteristic of this pattern.

Seeing only the sequence of distance from each part of the body to the Hip-Center does not provide enough information about the cause of the anomaly in the pattern. For this reason in Figure 13a, the Skeleton of the person is shown and in Figure 13b, the path of the Skeleton. It is observed that indeed at the beginning of the trajectory the position of the legs is narrow, where this characteristic of a Spastic Diplegic type is typical. This abnormality in normal gait may be due to a user's initial poor position or noise problems in the person's Skeleton, causing the network to incorrectly classify the sequence. 

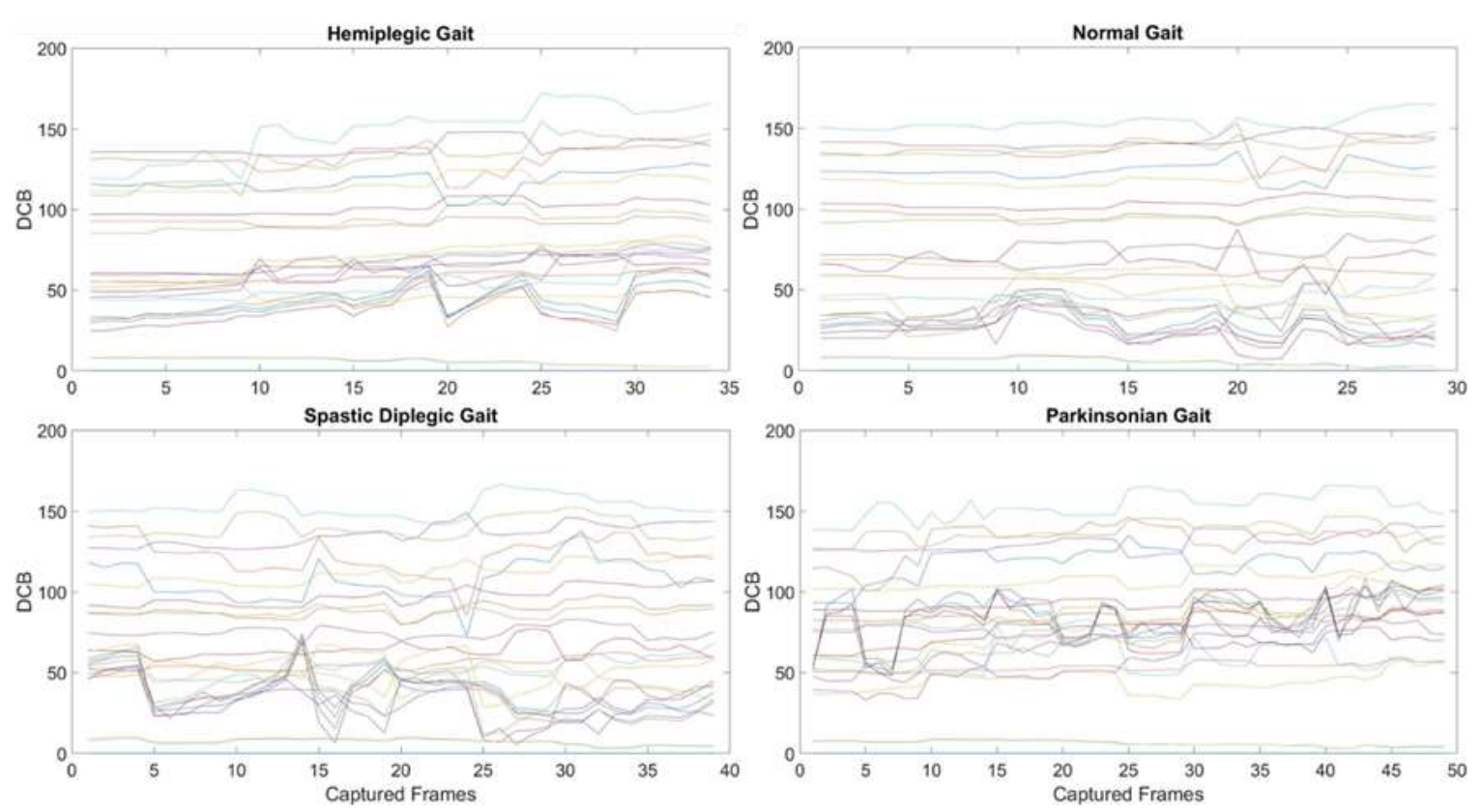

Figure 11. Sequences correctly classified

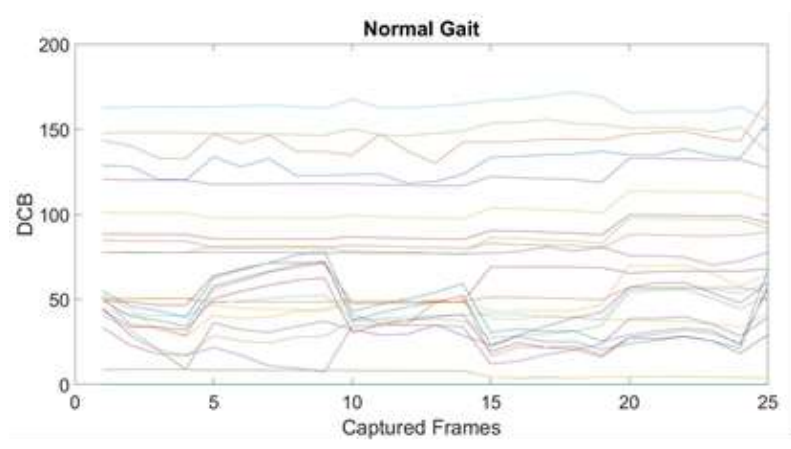

Figure 12. Wrong classification of the network

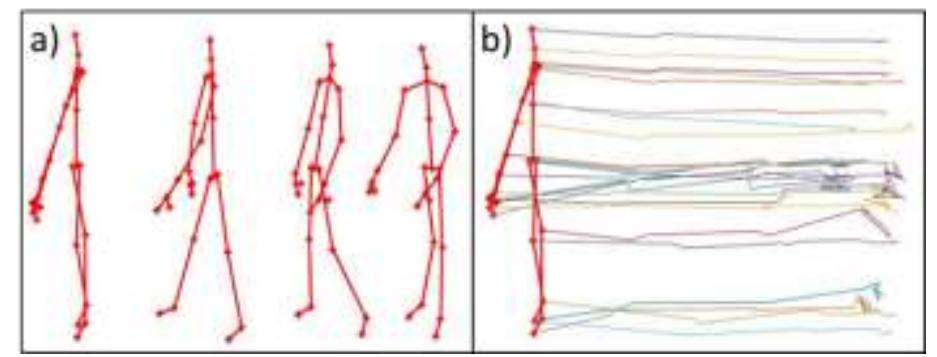

Figure 13. Skeleton incorrect classification

\subsection{Graphic user interface}

A graphic user interface is designed, in order to facilitate, organize and concatenate the processes presented in the paper. The first part of the interface is responsible for the acquisition stage of the database Figure 14. The first step in the acquisition of the Skeleton to calculate the databases is to select from the list what type of gait the person has. After clicking on "Acquire", the Kinect will be activated and once the person's Skeleton is detected for the first time, the button that says "Walk" will change to green indicating the user can start walking Figure 14(a). Once the person leaves the scene, in figure 14(b), the simulation of the gait of the user's Skeleton will be displayed, after which the 4 databases are calculated, plotted and stored Figure 14(c). 
DataAcquisition Train and Test Diagnosis

a) Type Of Gait

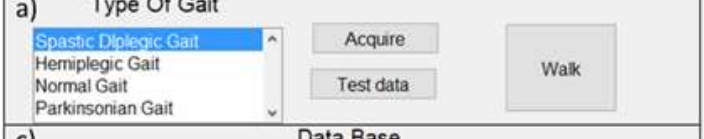

c)

Parkinsonian Gait

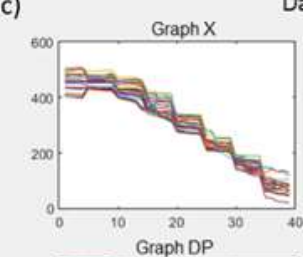

Graph DP

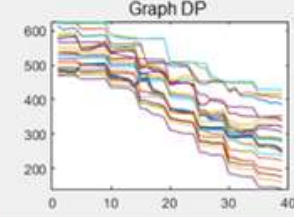

b)

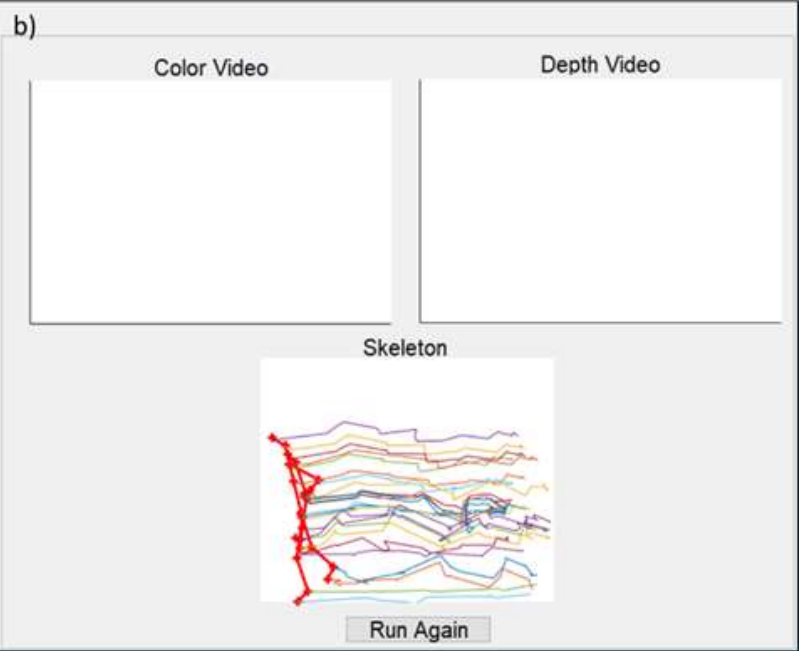

Figure 14. Acquisition of data in the graphic user interface

Once the database is acquired, the next step is to train the network. In Figure 15, the section of the interface responsible for this stage is shown. First, it must be selected in the list of Figure 15(a), with what type of database it is wanted to train the network, then, various parameters must be set: name of the network, percentage of the database that will be for training, number of categories, number of features, hidden units, size of the batch and the number of training epochs. Once a value is selected for each of these parameters, in Figure 15(b), it must be clicked on the train button. Once the training is finished, click on the test button. In Figure 15(c), an example of one of the sequences of the base to be trained, the size of the training and test sequences be shown. In addition, if the user wishes to see in more detail the tests of the trained network and observe the percentages of accuracy of the network in each category, they can do so by clicking on the "Confusion Matrix" button.

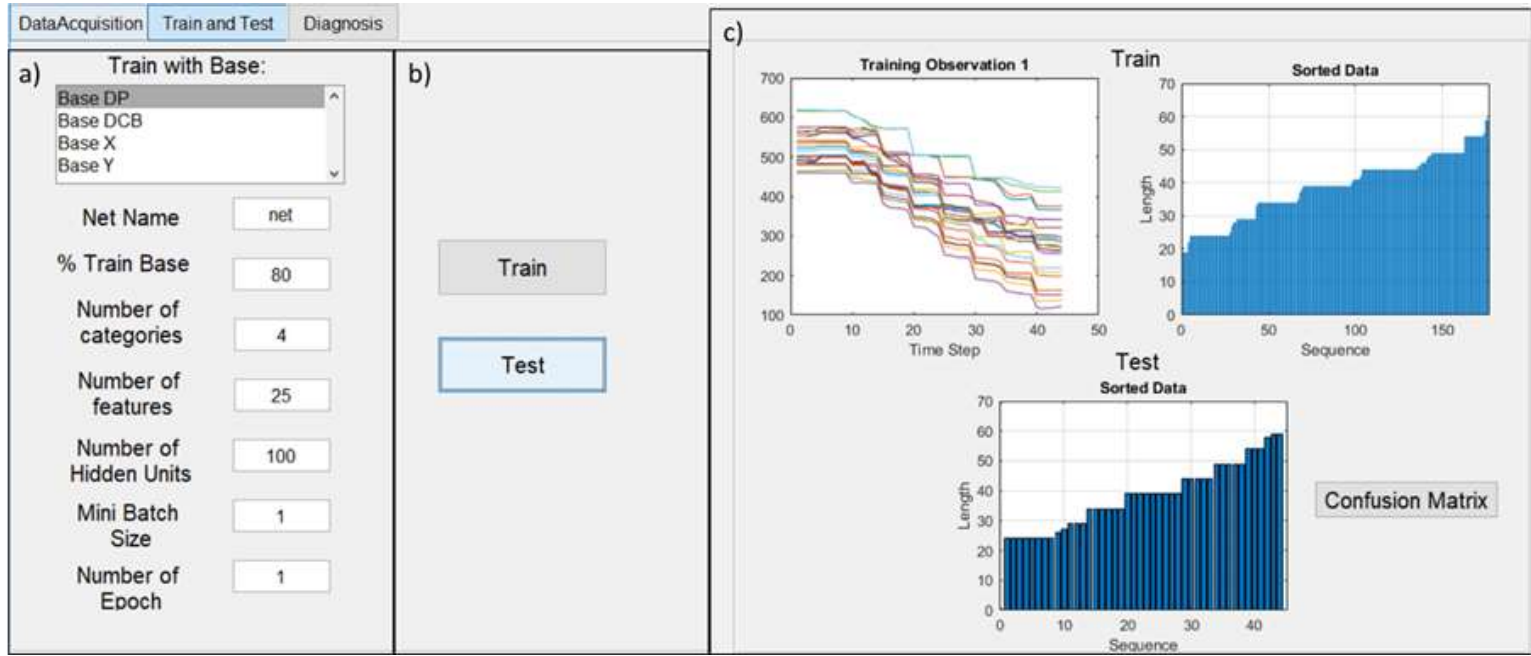

Figure 15. Train and Test in the graphic user interface

The last part of the interface is shown in Figure 16. This section focuses on testing the trained networks and showing the categories in which the input sequences are classified. The first step is shown in Figure 16a, the type of database is selected in the list, and then the name of the network trained with that database is chosen. The tests can be done in two ways, the first by clicking on the "Acquire" button, the Kinect will be activated and, as in Figure 14(a), will indicate the user when to start walking. Once the person is not detected, the simulation of the user's gait with the Skeleton will be generated in Figure 16(b), then in Figure 16(c) the category in which the input sequence was classified is displayed in 
green. The other option to perform the tests is by clicking on the "open base data" button of Figure 16(a), this will show a panel in this same section, the user must click on the pattern, then click on the "Open base" button, and a text will indicate how many videos it has available to test. Select a value between 1 and the indicated value of available data, if desired, can click on the radio button "Show Skeleton", to see, in Figure 16(b), the gait of the selected case. By clicking on the "run" button, the network will classify the selected pattern in one of the four categories of Figure 16(c). In the case of "Acquire", once the network classifies the sequence, the user can feed the databases of each walking pattern, by clicking on one of the buttons in the section of Figure 16(c).

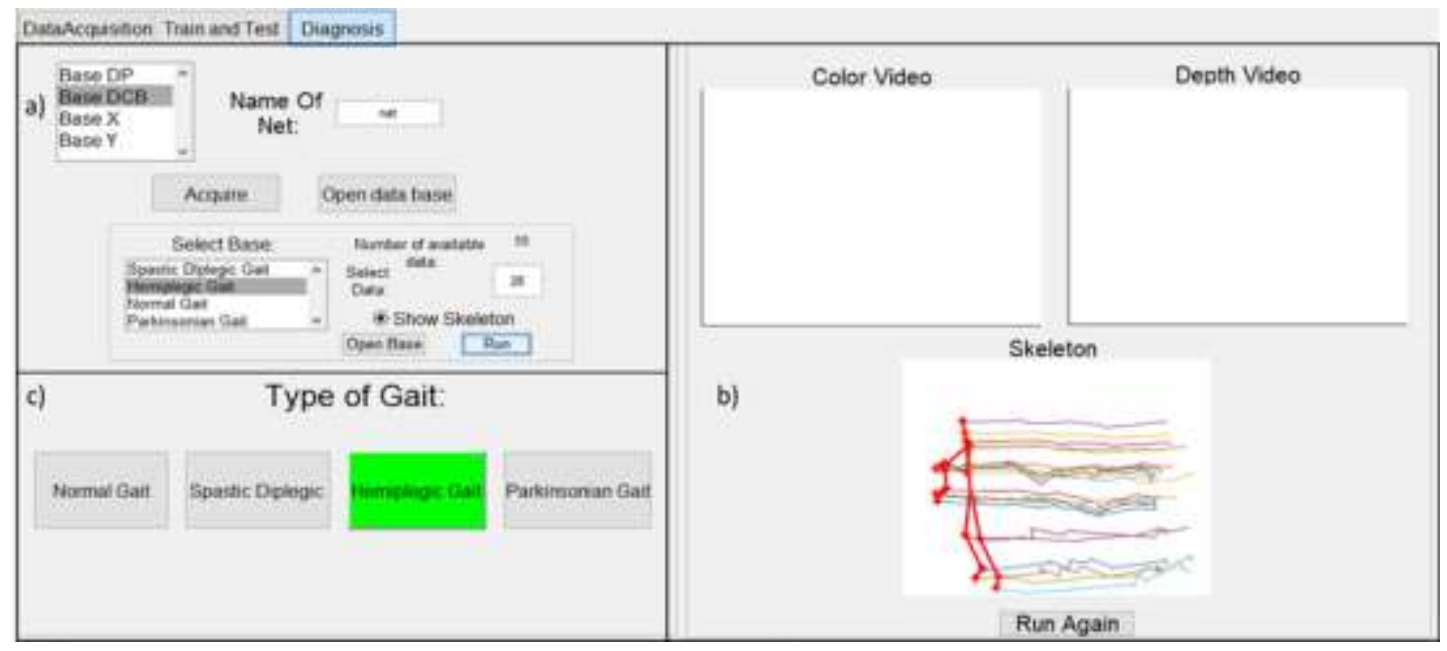

Figure 16. Diagnosis in the graphic user interface

\subsection{Graphic user interface}

An important step in systems that implement deep learning algorithms is to know the time it takes the network to classify the sequences. In this way, it can be known if it is viable for applications in real-time and that, instead of causing delays in the processes, they are optimized. Table 2 shows the times it takes for the trained network with the DCB database to classify a single sequence. It should be noted that the tests were performed on a computer with an NVIDIA $1050 \mathrm{Ti}$ GPU, 16 GB of RAM and an eighth-generation i7 processor.

Table 2. Processing times

\begin{tabular}{cc}
\hline Type of Gait & Time $(\mathrm{s})$ \\
\hline Normal & 0.0209 \\
Spastic Diplegic & 0.0213 \\
Hemiplegic & 0.0210 \\
Parkinsonian & 0.0228 \\
\hline Average time: & 0.0215 \\
\hline
\end{tabular}

\section{CONCLUSION}

Based on the results of the networks trained with each of the 4 bases, having a reference point in the body of the person during the walk allows the LSTM to discriminate the features of each type of gait pattern more accurately, in this case obtaining a $98.1 \%$ accuracy in the tests of the network. Identifying that the proposed system for the detection of abnormalities gait can be a useful support tool for doctors who need to make a diagnosis of this type. The proposed method and graphic user interface, apart from the Kinect sensor and a computer, do not require intrusive devices that may affect the person's gait pattern. Allowing it to be a tool that can serve as a support to physicians in the diagnosis of neurological diseases related to the gait patterns worked. The processing times show that, once the sequence to be classified is entered, the times will not vary significantly depending on the type of gait. In addition, having an average time of $0.0215 \mathrm{~s}$ in the classification will not significantly affect the patient's diagnostic processes. 
To improve the accuracy of the network, it is necessary to test other methods to generate databases from the coordinates of the 25 points of the Skeleton, either changing the reference point in the body, combining the features of current databases or testing other mathematical methods. Another important point is to increase the database to train new networks or retrain existing ones. An interesting starting point for future developments, given the results obtained, is to include additional gait patterns to train the network, such as Myopathic Gait, Ataxic Gait or Sensory Gait. This would allow the system to address most of the current abnormalities that may occur in the walk of people

\section{ACKNOWLEDGEMENTS}

The authors are grateful to the Nueva Granada Military University for the support given in the development of this work.

\section{REFERENCES}

[1] S. Valverde, et al., "Improving automated multiple sclerosis lesion segmentation with a cascaded 3D convolutional neural network approach," NeuroImage, Vol. 155, pp.159-168, 2017. doi: 10.1016/j.neuroimage.2017.04.034.

[2] J. Schmidhuber, "Deep learning in neural networks: An overview", Neural Networks, Volume 61, January 2015, pp. 85-117.

[3] A. Krizhevsky, I. Sutskever, and G. E. Hinton, "Imagenet classification with deep convolutional neural networks", In Advances in neural information processing systems, 2012, pp. 1097-1105.

[4] Russakovsky, O., Deng, J., Su, H., et al. "ImageNet Large Scale Visual Recognition Challenge." International Journal of Computer Vision (IJCV). Vol 115, Issue 3, 2015, pp. 211-252.

[5] Shahram Taheri, Önsen Toygar, On the use of DAG-CNN architecture for age estimation with multi-stage features fusion, Neurocomputing, Volume 329, 2019, Pages 300-310, ISSN 0925-2312, https://doi.org/10.1016/j.neucom.2018.10.071.

[6] César Giovany Pachón Suescún, Javier Orlando Pinzón Arenas, Robinson Jiménez Moreno. Detection of Scratches on Cars by Means of CNN and R-CNN. International Journal on Advanced Science, Engineering and Information Technology (IJASEIT), ISSN : 2088-5334. Vol. 9 (2019) No. 3pages: 745-752. DOI:10.18517/ijaseit.9.3.6470

[7] P. Moeskops, M. A. Viergever, A. M. Mendrik, L. S. de Vries, M. J. N. L. Benders and I. Išgum, "Automatic Segmentation of MR Brain Images With a Convolutional Neural Network," in IEEE Transactions on Medical Imaging, vol. 35, no. 5, pp. 1252-1261, May 2016.

[8] R. Torres, et al., "Diagnosis of the corporal movement in Parkinson's Disease using Kinect Sensors," In World Congress on Medical Physics and Biomedical Engineering, 2015, pp. 1445-1448. doi: 10.1007/978-3-319-193878352.

[9] Microsoft, "Kinect for Windows", [online], Available at: https://developer.microsoft.com/en-us/windows/kinect. Consult date: Aug 27, 2019.

[10] A. Zhao, L. Qi, J. Dong, and H. Yu., "Dual channel LSTM based multi-feature extraction in gait for diagnosis of Neurodegenerative diseases," Knowledge-Based Systems, Vol. 145, pp.91-97. $2018 . \quad$ doi: 10.1016/j.knosys.2018.01.004.

[11] Meng Chen, Bufu Huang and Yangsheng Xu, "Intelligent shoes for abnormal gait detection," 2008 IEEE International Conference on Robotics and Automation, Pasadena, CA, 2008, pp. 2019-2024.

[12] D. Arifoglu, and A. Bouchachia., "Activity Recognition and Abnormal Behaviour Detection with Recurrent Neural Networks," Procedia Computer Science, Vol. 110, pp.86-93, 2017. doi: 10.1016/j.procs.2017.06.121.

[13] Y. Hou, Z. Li, P. Wang, and W. Li., "Skeleton optical spectra based action recognition using convolutional neural networks," IEEE Transactions on Circuits and Systems for Video Technology, 2016, pp. 807-811. doi: 10.1109/TCSVT.2016.2628339.

[14] E.P. Ijjina, and K.M. Chalavadi., "Human action recognition using genetic algorithms and convolutional neural networks," Pattern recognition, Vol. 59, pp.199-212, 2016. doi: 10.1016/j.patcog.2016.01.012.

[15] K.K Reddy, and M. Shah., "UCF50 action recognition dataset," date accessed, Vol. 29, no. 7, 2015.

[16] Y. Chen, K. Zhong, J. Zhang, Q. Sun, and X. Zhao., "Lstm networks for mobile human activity recognition," In International Conference on Artificial Intelligence: Technologies and Applications. ICAITA. 2016, pp. 50-13. doi: 10.2991/icaita-16.2016.13.

[17] J. Liu, A. Shahroudy, D. Xu, and G. Wang., "Spatio-temporal 1stm with trust gates for 3d human action recognition," In European Conference on Computer Vision, Springer, Cham, 2016, pp. 816-833. doi: 10.1007/9783-319-46487-9 50.

[18] L. Xia, C. Chen and J. K. Aggarwal, "View invariant human action recognition using histograms of 3D joints," 2012 IEEE Computer Society Conference on Computer Vision and Pattern Recognition Workshops, Providence, RI, 2012, pp. 20-27.

[19] World Health Organization., "Neurological disorders: public health challenges," World Health Organization, 2016.

[20] C. Olah., "Understanding lstm networks", [online], Available at: https://colah.github.io/posts/2015-08Understanding-LSTMs. Consult date: Aug 27, 2019.

[21] A. Karpathy, J. Johnson, and L. Fei-Fei., "Visualizing and understanding recurrent networks," 2015, arXiv preprint arXiv:1506.02078. 
[22] S. Hochreiter, and J. Schmidhuber., "Long short-term memory," Neural computation, vol. 9, no. 8, pp.1735-1780, 1997. doi: 10.1162/neco.1997.9.8.1735

[23] M.R. Lim, R.C. Huang, A. Wu, F.P. Girardi, and F.P. Cammisa Jr., "Evaluation of the elderly patient with an abnormal gait," JAAOS-Journal of the American Academy of Orthopaedic Surgeons, vol. 15, no. 2, pp.107-117. 2007. doi: 10.5435/00124635-200702000-00005.

[24] Stanford Medicine, "Gait Abnormalities", [online], https://stanfordmedicine25.stanford.edu/the25/gait.html. Consult Date: Aug 27, 2019.

[25] Y. Bengio, R. Ducharme, P. Vincent, and C. Jauvin., "A neural probabilistic language model," Journal of machine learning research, pp.1137-1155, 2003.

[26] D. Masters, C. Luschi., "Revisiting Small Batch Training for Deep Neural Networks," 2018, arXiv preprint arXiv:1804.07612.

\section{BIOGRAPHIES OF AUTHORS}
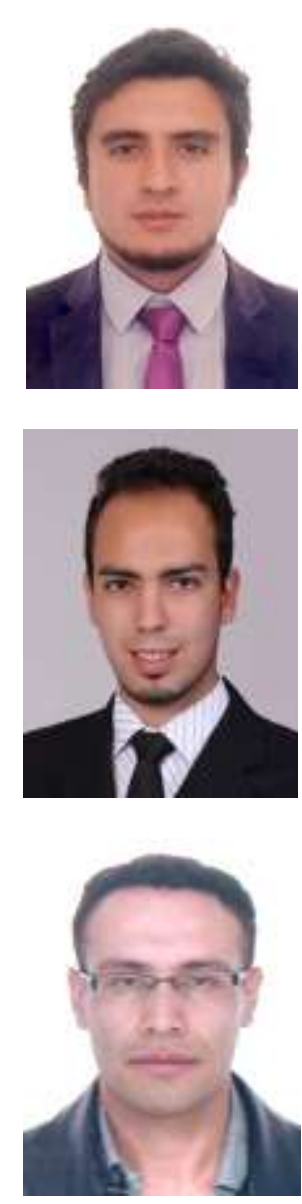

César Giovany Pachón Suescún was born in Bogotá, Colombia, in 1996. He received his degree in Mechatronics Engineering from the Pilot University of Colombia in 2018. Currently, he is studying his Master's degree in Mechatronics Engineering and working as Research Assistant at the Nueva Granada Military University with an emphasis on Robotics and Machine Learning. E-mail: u3900259@unimilitar.edu.co

Javier Orlando Pinzón Arenas was born in Socorro-Santander, Colombia, in 1990. He received his degree in Mechatronics Engineering (Cum Laude) in 2013, Specialization in Engineering Project Management in 2016, and M.Sc. in Mechatronics Engineering in 2019, at the Nueva Granada Military University-UMNG. He has experience in the areas of automation, electronic control and machine learning. Currently, he is studying a Ph.D. in Applied Sciences and working as Graduate Assistant at the UMNG with emphasis on Robotics and Machine Learning. E-mail: u3900231@unimilitar.edu.co

Robinson Jiménez Moreno was born in Bogotá, Colombia, in 1978. He received the Engineer degree in Electronics at the Francisco José de Caldas District University-UD-in 2002. M.Sc. in Industrial Automation from the Universidad Nacional de Colombia-2012 and Ph.D. in Engineering at the Francisco José de Caldas District University-2018. He is currently working as a Professor in the Mechatronics Engineering Program at the Nueva Granada Military University-UMNG. He has experience in the areas of Instrumentation and Electronic Control, acting mainly in Robotics, control, pattern recognition, and image processing. E-mail: robinson.jimenez@unimilitar.edu.co 